\title{
Technical difficulty grade score for the laparoscopic approach of rectal cancer
}

\author{
A single institution pilot study
}

\author{
A. A. F. A. Veenhof - A. F. Engel - D. L. van der Peet • \\ C. Sietses - W. J. H. J. Meijerink • \\ E. S. M. de Lange-de Klerk • M. A. Cuesta
}

Accepted: 13 December 2007 / Published online: 9 January 2008

(C) The Author(s) 2007

\begin{abstract}
Introduction We aimed to categorize laparoscopic rectal resections according to technical difficulty to standardize learning purposes and stratify results, making future studies more comparable.

Materials and methods Fifty patients undergoing a laparoscopic total mesorectal excision were prospectively followed. Four preoperatively known facts (gender, body mass index (BMI), tumor localization, and preoperative radiation therapy) were compared to four operative outcomes (operation time, blood loss, a visual analogue score (VAS) for difficulty rewarded by the surgeon, and oncological radicality of the procedure).

Results Operating time for male and female patients was 257 vs. $245 \mathrm{~min}(P=0.229)$, blood loss was 300 vs. $300 \mathrm{ml}$ $(P=0.309)$, the VAS was 8 vs. $6(P<0.001)$, and radicality was $93 \%$ vs. $91 \%(P=0.806)$. Operating time was 215,250 , and $305 \mathrm{~min}$ for high, mid, and low tumors (Spearman $-0.44 ; P=0.02)$, respectively. Blood loss was 105,300 , and $600 \mathrm{ml}$ (Spearman $-0.38 ; P=0.01$ ). Lower tumors were rewarded a higher VAS (Spearman $-0.57 ; P<0.001$ ) and
\end{abstract}

A. A. F. A. Veenhof · D. L. van der Peet · C. Sietses •

W. J. H. J. Meijerink $\cdot$ M. A. Cuesta $(\triangle)$

Departments of Surgery, Vrije Universiteit Medical Center,

Postbus 7057, 1007 MB Amsterdam, The Netherlands

e-mail:ma.cuesta@vumc.nl

\section{A. F. Engel}

Department of Surgery, Zaans Medical Center,

Zaandam, The Netherlands

E. S. M. de Lange-de Klerk

Department of Clinical Epidemiology and Biostatistics,

VU University Medical Center,

Amsterdam, The Netherlands were less often radically resected (Spearman $0.32 ; P=$ 0.026). Operating time for irradiated and nonirradiated patients was 277 vs. $225 \min (P=0.008)$, blood loss was 500 vs. $150 \mathrm{ml}(P=0.006)$, the VAS was 7 vs. $5(P<0.001)$, and radicality was $79 \%$ vs. $100 \%(P=0.046)$. Operating time was 240 min for BMI 25-30 and 253 min for BMI $>30$ (Spearman 0.13; $P=0.391$ ). Blood loss was $150 \mathrm{ml}$ for BMI 25-30 and $500 \mathrm{ml}$ for BMI $>30$ (Spearman 0.38; $P=0.01$ ). Higher BMIs were rewarded a higher VAS (Spearman 0.06; $P=0.704)$. BMI had no correlation to radicality of the procedure (Spearman $-0.12 ; P=0.402$ ). There was an association between technical difficulty score and operation time $(P=0.007)$, blood loss $(P<0.001)$, VAS $(P<0.001)$, and radicality of surgery $(P=0.043)$.

Conclusion Laparoscopic surgery in male, irradiated, and obese patients with lower tumors seemed more difficult. A categorization according to technical difficulty, to preoperatively predict difficulty of the procedure, was found feasible.

Keywords Total mesorectal excision · Learning curve · Laparoscopy $\cdot$ Rectal cancer

\section{Introduction}

Presently, little distinction is made regarding the technical difficulty grade in laparoscopic rectal surgery. Most studies only refer to the localization of the tumor as a degree for technical difficulty of the operation, leaving many other factors unaccounted for. The distinct difference in male and female pelvic anatomy makes female patients theoretically more suitable for laparoscopic rectal surgery, as there is usually more opportunity to maneuver in the wider pelvic 
structure. Also, the body mass index (BMI) is a factor to consider when referring to the technical difficulty in laparoscopic rectal surgery, as is the presence of preoperative radiation therapy (RHT), especially when a long delay between radiation therapy and surgery is applied. Although the tumor size is often reduced after a long delay to surgery, the surrounding tissue is frequently fibrotic and difficult to handle.

It may therefore be useful to define the technical difficulty of laparoscopic rectal resections to improve and standardize education for others, to counsel the learning process, for self-evaluation, and to stratify results, making different future publications regarding laparoscopic rectal surgery more comparable.

The aim of this study was to see if laparoscopic rectal surgery could be categorized according to their technical difficulty degree on the bases of preoperatively known facts, such as gender, body mass index, localization of the tumor, and preoperatively administered radiation therapy.

\section{Materials and methods}

\section{Patients}

Eight factors were prospectively evaluated in 50 consecutive patients who underwent a laparoscopic resection for rectal cancer in the VU University Medical Center. Patients were operated on between April 2002 and November 2005 by the same surgeon and surgical team. Patients who had previous abdominal surgery through a median laparotomy were excluded from this study, as were patients undergoing palliative surgery or surgery for benign tumors.

All patients received the same pretreatment workup, including a colonoscopy with biopsies for rectal examination: rectal ultrasound and magnetic resonance imaging of the rectum for local staging, and X-thorax, computed tomography scan of the abdomen, and cinoembryonic antigen examination for dissemination status. Tumors had to be located within $17 \mathrm{~cm}$ of the anal verge, measured by rigid scope following colonoscopy with rectal ultrasound, and were defined according to Goligher [1]: $7 \mathrm{~cm}$ or less from the anal verge, low rectum; over $7 \mathrm{~cm}$ but less than or equal to $12 \mathrm{~cm}$, mid rectum; over $12 \mathrm{~cm}$ but less than or equal to $17 \mathrm{~cm}$, high rectum.

Before each operation, the following factors were taken into consideration: male or female pelvis, body mass index, localization of the tumor in centimeter from the dentate line, and whether preoperative radiation therapy was administered. These factors were subsequently compared to the following operative outcomes: length of operating time, blood loss, whether the tumor was radically resected (circumferential margin $>2 \mathrm{~mm}$ ), and a visual analogue score (VAS) for difficulty from the surgeon, ranging from one through ten, with score one being the easiest laparoscopic operation ever performed by the surgeon and score ten being a conversion to laparotomy.

We consequently reviewed whether the preoperatively determined parameters could predict the difficulty grade of the operation, expressed in terms of the operative outcomes. On the basis of postoperative outcomes regarding the preoperatively known patient characteristics, we created a point system as a reference for technical difficulty grade for laparoscopic total mesorectal excisions (TMEs).

\section{Collected data}

- Patient data: gender, age, body mass index, localization of the tumor, and preoperative radiation therapy

- Operative data: operating time, blood loss, VAS for difficulty by the surgeon, and oncological radicality of the procedure

\section{Statistical analysis}

Statistical analysis was performed using the SPSS software package (SPSS 11.5 for Windows; SPSS, Chicago, IL, USA). Medians and interquartile ranges were calculated and subsequently depicted when appropriate. Spearman's correlation, the Mann-Whitney $U$ test, the Kruskal-Wallis test, chi-squared test, or Fisher's exact test were applied when appropriate for group comparisons. Significance was set at $P<0.05$.

\section{Results}

\section{Patient characteristics}

Fifty patients underwent a laparoscopic TME with curative intent. In $34(68 \%)$ of the patients, a TME was performed, followed by a primary anastomosis. In ten of these patients, a colo-anal anastomosis was performed because of very distal cancer in the rectum. The other $16(32 \%)$ patients underwent an abdominoperineal resection (APR).

Patient characteristics are depicted in Table 1. The group included 28 men and 22 women with a median age of 67 years (interquartile range 58-75). The median body mass index was 25 (interquartile range 23-27). The median distance between tumor and anal verge was $8.0 \mathrm{~cm}$ (range 2-17). All 26 patients with mid and low rectal tumors (52\%) underwent neo-adjuvant radiation therapy, including two patients $(4 \%)$ who also received neo-adjuvant chemotherapy. In our institution, a delay of $4-6$ weeks is maintained between radiation therapy and surgery. The 
Table 1 Patient and tumor characteristics

\begin{tabular}{ll}
\hline Variable & $\begin{array}{l}\text { Laparoscopic } \\
\text { TME }(n=50)\end{array}$ \\
\hline Gender, number (\%) & \\
Male & $28(56 \%)$ \\
Female & $22(44 \%)$ \\
Age-median years (interquartile range) & $67(58-75)$ \\
BMI-median (interquartile range) & $25(23-27)$ \\
Median distance from anal verge to tumor, & $8.0(2-17)$ \\
cm (full range) &
\end{tabular}

median delay between the last dose of radiation therapy and surgery was 44.5 days (interquartile range 9-52).

Male vs. female pelvis

Median operating time was $257 \mathrm{~min}$ (interquartile range 223-305) for male patients and $245 \mathrm{~min}$ (interquartile range 200-280) for female patients $(P=0.229)$. Median blood loss was $300 \mathrm{ml}$ (interquartile range 200-700) for male patients and $300 \mathrm{ml}$ (interquartile range 100-600) for female patients $(P=0.309)$. The median VAS for difficulty rewarded by the surgeon was 8 (interquartile range 7-9) for male patients and 6 (interquartile range 3-7) for female patients $(P<0.001)$. Radical excision of the tumor in male and female patients was achieved in $93 \%$ and $91 \%$, respectively $(P=0.806$; Table 2$)$.

\section{Tumor localization}

Scatter graphs with the Spearman correlation for tumor localization vs. operative outcomes are depicted in Figs. 1, 2, and 3. Median operating time was $215 \mathrm{~min}$ (interquartile range 205-236.5) for high rectal cancer, $250 \mathrm{~min}$ (interquartile range 221-265) for mid rectal cancer, and $305 \mathrm{~min}$ (interquartile range 244-320) for low rectal cancer. There was a significant association between operating time and localization of the tumor: the lower the tumor, the longer the operating time (Spearman $-0.44 ; P=0.002$ ). Median

Table 2 Male vs. female results

\begin{tabular}{llll}
\hline Variable & $\begin{array}{l}\text { Male } \\
(n=28)\end{array}$ & $\begin{array}{l}\text { Female } \\
(n=22)\end{array}$ & Significance \\
\hline $\begin{array}{l}\text { Length of operation in } \\
\text { minutes - median } \\
\text { (interquartile range) }\end{array}$ & $\begin{array}{c}257 \\
(223-305)\end{array}$ & $\begin{array}{c}(245 \\
(200-280)\end{array}$ & $P=0.229$ \\
$\begin{array}{c}\text { Blood loss in milliliter- } \\
\text { median (interquartile range) }\end{array}$ & 300 & 300 & $P=0.309$ \\
$\begin{array}{c}\text { VAS difficulty score- } \\
\text { median (interquartile range) }\end{array}$ & $8(7-9)$ & $6(3-7)$ & $P<0.001$ \\
\begin{tabular}{c} 
Radical excision tumor \\
\hline
\end{tabular} & $93 \%$ & $91 \%$ & $P=806$ \\
\hline
\end{tabular}

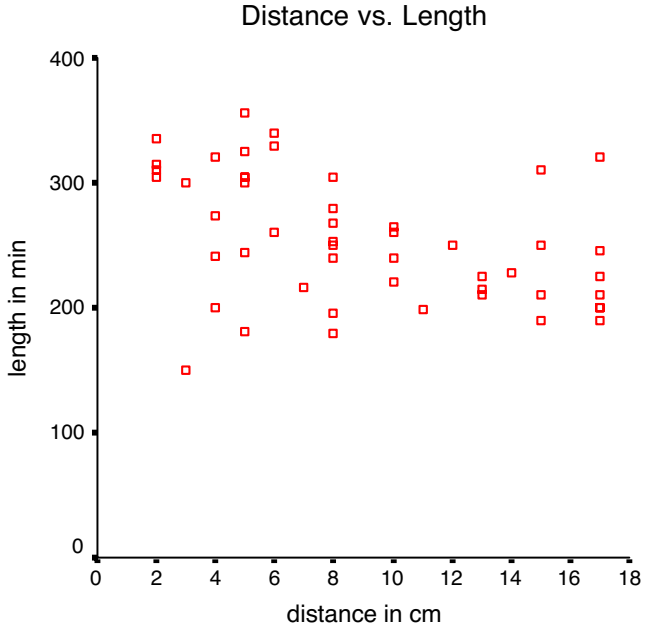

Fig. 1 Tumor distance vs. length of the operation. Correlation coefficient, $-0.44(P=0.002)$

blood loss was $105 \mathrm{ml}$ (interquartile range 50-225) for high rectal cancer, $300 \mathrm{ml}$ (interquartile range 200-500) for mid rectal cancer, and $600 \mathrm{ml}$ (interquartile range 225-900) for low rectal cancer. There was a significant association between blood loss and localization of the tumor: the lower the tumor, the more blood loss (Spearman $-0.38 ; P=0.01$ ). There was a significant association between VAS for difficulty and localization of the tumor: the lower the tumor, the higher the VAS for difficulty (Spearman -0.57 ; $P<0.001)$. In lower localized tumors, a radical excision was less often achieved (Spearman 0.32; $P=0.026$ ).

Preoperative radiation therapy

Median operating time was $225 \mathrm{~min}$ (interquartile range 205-252) for patients without RHT and $277 \mathrm{~min}$ (interquartile range 240-314) for patients with RHT $(P=0.008)$. Median blood loss was $150 \mathrm{ml}$ (interquartile range 50-350)

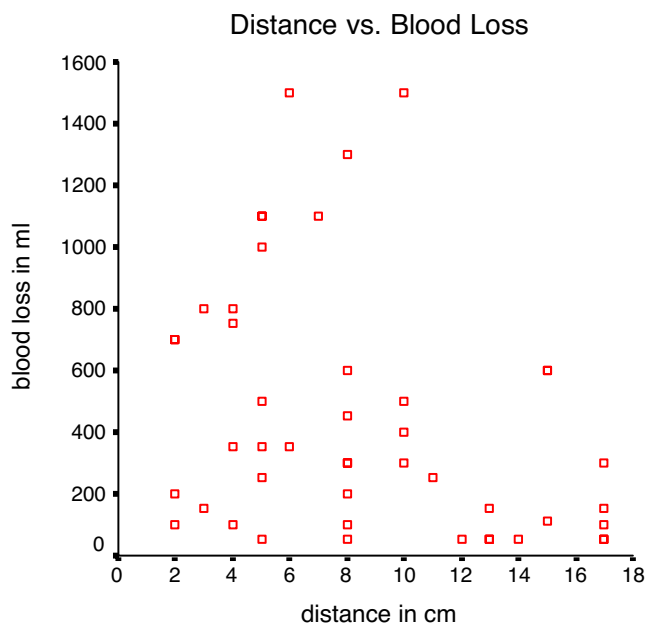

Fig. 2 Tumor distance vs. blood loss. Correlation coefficient, -0.38 $(P=0.01)$ 
Distance vs. Surgeon Score

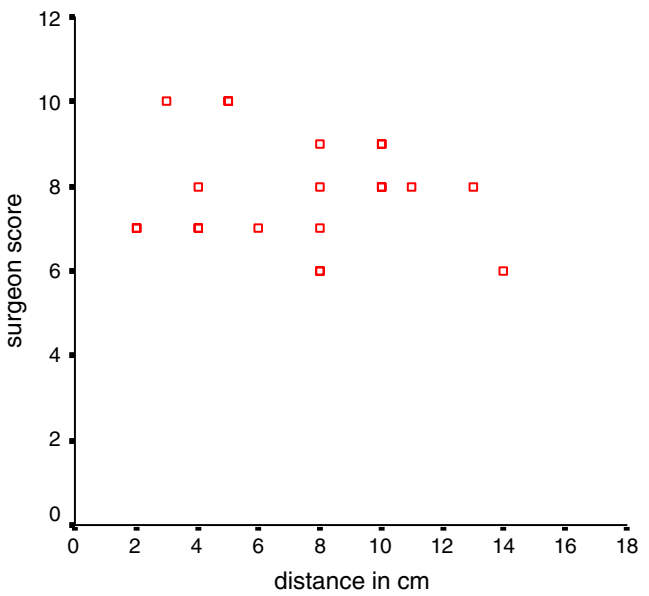

Fig. 3 Tumor distance vs. surgeon score. Correlation coefficient, $-0.57(P<0.001)$

for patients without RHT and $500 \mathrm{ml}$ (interquartile range $250-800)$ for patients with RHT $(P=0.006)$. The median VAS for difficulty rewarded by the surgeon was 5 (interquartile range 3-8) for patients without RHT and 7 (interquartile range 7-8) for patients with RHT $(P<0.001)$. Radical excision of the tumor in nonirradiated and irradiated patients was achieved in $100 \%$ and $79 \%$, respectively $(P=0.046$; Table 3$)$.

Body mass index

Scatter graphs with the Spearman correlation for BMI vs. operative outcomes are depicted in Figs. 4, 5, and 6. Median operating time was $240 \mathrm{~min}$ (interquartile range 200-305) for BMI 25-30 patients and 253 min (interquartile range 221-305) for BMI $>30$ patients. There was no significant association between operating time and BMI (Spearman 0.13; $P=0.391$ ). Median blood loss was $150 \mathrm{ml}$ (interquartile range 75-300) for BMI 25-30 patients and $500 \mathrm{ml}$ (interquartile range 225-725) for BMI $>30$ patients. There was a significant association between blood loss and BMI: the higher the BMI, the more blood was lost

Table 3 RHT vs. no RHT results

\begin{tabular}{llll}
\hline Variable & $\begin{array}{l}\text { RHT } \\
(n=26)\end{array}$ & $\begin{array}{l}\text { No RHT } \\
(n=24)\end{array}$ & Significance \\
\hline $\begin{array}{l}\text { Length of operation in } \\
\text { minutes - median } \\
\text { (interquartile range) }\end{array}$ & $\begin{array}{c}277 \\
(240-314)\end{array}$ & $\begin{array}{c}225 \\
(205-252)\end{array}$ & $P=0.008$ \\
$\begin{array}{c}\text { Blood loss in milliliter- } \\
\text { median (interquartile range) }\end{array}$ & 500 & 150 & $P=0.006$ \\
$\begin{array}{l}\text { VAS difficulty score- } \\
\text { median (interquartile range) }\end{array}$ & $7(7-8)$ & $5(3-8)$ & $P<0.001$ \\
\begin{tabular}{c} 
Radical excision tumor \\
\hline
\end{tabular} & $79 \%$ & $100 \%$ & $P=0.046$ \\
\hline
\end{tabular}

BMI vs. Length

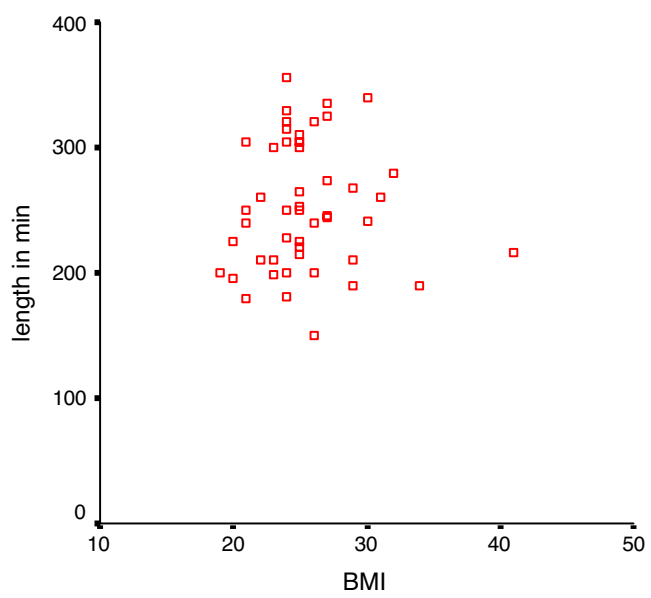

Fig. 4 BMI vs. length of the operation. Correlation coefficient, 0.13 $(P=0.391)$

(Spearman 0.38; $P=0.01$ ). There was no significant association between VAS for difficulty and BMI (Spearman 0.06; $P=0.704)$. Also, no association between radical excision of the tumor and BMI was found (Spearman $-0.12 ; P=0.402)$.

Point-score system

On the basis of the above depicted postoperative outcomes regarding the preoperatively known patient characteristics, we created an artificial point system as a reference for technical difficulty grade for laparoscopic TMEs (Table 4): two points for a male patient and one point for a female patient; in addition, 15, 10, and 5 points for a tumor located in the low, mid, or high rectum, respectively; in addition, ten points for patients with a BMI $>30$ and five points for patients with a BMI 25-30; likewise, ten points for patients who received preoperative radiation therapy (Table 4).

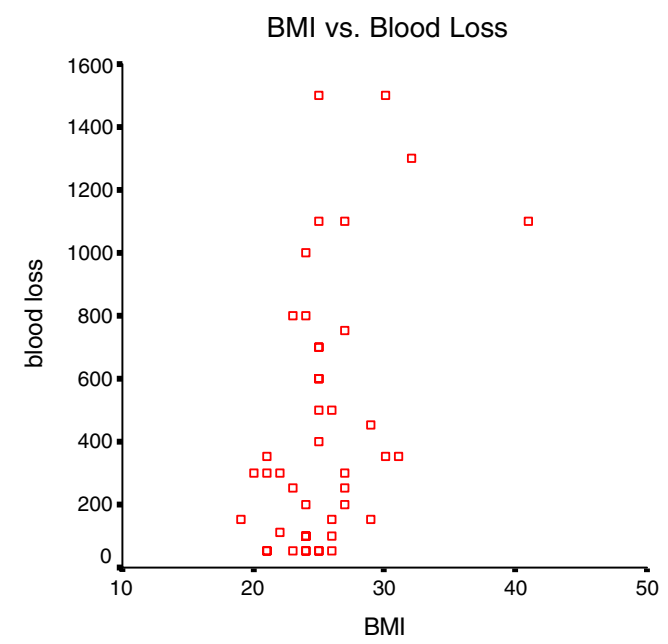

Fig. 5 BMI vs. blood loss. Correlation coefficient, $0.38(P=0.01)$ 
BMI vs. Surgeon Score

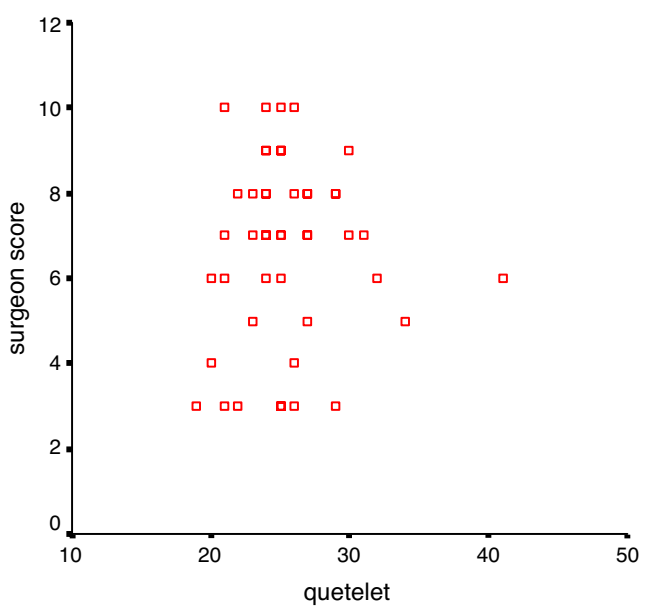

Fig. $6 \mathrm{BMI}$ vs. surgeon score. Correlation coefficient, $0.06(P=$ $0.704)$

Theoretically, patient scores between 6 and 37 could be rewarded. "Low-score patients" were patients defined by scores 6-11, "medium-score patients" were patients defined by scores $12-24$, and "high-score patients" were defined by scores $25-37$. We subsequently evaluated whether this point system was validated when comparing this "technical difficulty grade" to objective blood loss, operating time, VAS for difficulty by the surgeon, and oncological radicality of the procedure.

Low, medium, and high technical difficulty grade score

Low technical difficulty grade laparoscopic TMEs (score 6-11) had a median operating time of $220 \mathrm{~min}$ (interquartile range 200-250) with a median blood loss of $50 \mathrm{ml}$ (interquartile range 50-140), a median VAS for difficulty by the surgeon of 3 (interquartile range 3-4), and a radical excision in $100 \%$. Medium technical difficulty grade

Table 4 Difficulty score index

\begin{tabular}{ll}
\hline Variable & Points \\
\hline Gender & \\
Male & 2 \\
Female & 1 \\
Preoperative RHT & \\
Yes & 10 \\
No & 0 \\
Tumor localization & \\
Low rectum $(0-7 \mathrm{~cm})$ & 15 \\
Mid rectum $(>7-12 \mathrm{~cm})$ & 10 \\
High rectum $(>12-17 \mathrm{~cm})$ & 5 \\
BMI & \\
$<25$ & 0 \\
$25-30$ & 5 \\
$>30$ & 10 \\
\hline
\end{tabular}

laparoscopic TMEs (score 12-24) had a median operating time of $248 \mathrm{~min}$ (interquartile range 216-263) with a median blood loss of $300 \mathrm{ml}$ (interquartile range 225-375), a median VAS for difficulty by the surgeon of 8 (interquartile range $7-8$ ), and a radical excision in $100 \%$. High technical difficulty grade laparoscopic TMEs (score 25-37) had a median operating time of $290 \mathrm{~min}$ (interquartile range 241-317) with a median blood loss of $600 \mathrm{ml}$ (interquartile range $250-1,100$ ), a median VAS for difficulty by the surgeon of 8 (interquartile range 7-9), and a radical excision in $83 \%$. According to the Spearman correlation, there was a significant association between technical difficulty grade score and length of the operation $(P=$ $0.006)$, blood loss $(P<0.001)$, VAS for difficulty by the surgeon $(P<0.001)$, and oncological radicality of the procedure $(P=0.043$; Table 5$)$.

\section{Discussion}

The fact that a learning curve exists for different operations, especially in laparoscopic surgery, is generally well known and has been extensively published from the onset of laparoscopic surgery [2]. Some publications have suggested that the learning curve for colorectal laparoscopic surgery ranges from 11 to 80 procedures performed [3-5]. However, presently, no series trying to support and counsel surgeons by optimizing their learning curve have been published.

Geis et al. $[6,7]$ studied the relative difficulty of seven laparoscopic colonic procedures, differentiating in three complexity scales in laparoscopic colectomy: mobilization,

Table 5 Difficulty grade score

\begin{tabular}{|c|c|c|c|c|}
\hline Variable & $\begin{array}{l}\text { Low } \\
\text { difficulty } \\
\text { (6-11 } \\
\text { points) }\end{array}$ & $\begin{array}{l}\text { Medium } \\
\text { difficulty } \\
\text { (12-24 } \\
\text { points) }\end{array}$ & $\begin{array}{l}\text { High } \\
\text { difficulty } \\
\text { (25-37 } \\
\text { points) }\end{array}$ & Significance \\
\hline $\begin{array}{l}\text { Length of operation } \\
\text { in minutes- } \\
\text { median } \\
\text { (interquartile } \\
\text { range) }\end{array}$ & $\begin{array}{l}200 \\
(220-250)\end{array}$ & $\begin{array}{l}248 \\
(216-263)\end{array}$ & $\begin{array}{l}290 \\
(241-317)\end{array}$ & $P=0.006$ \\
\hline $\begin{array}{l}\text { Blood loss in } \\
\text { milliliter-median } \\
\text { (interquartile } \\
\text { range) }\end{array}$ & $\begin{array}{l}50 \\
(50-140)\end{array}$ & $\begin{array}{l}300 \\
(225-375)\end{array}$ & $\begin{array}{l}600 \\
(250-1,000)\end{array}$ & $P<0.001$ \\
\hline $\begin{array}{l}\text { VAS difficulty } \\
\text { score-median } \\
\text { (interquartile } \\
\text { range) }\end{array}$ & $3(3-4)$ & $8(7-8)$ & $8(7-9)$ & $P<0.001$ \\
\hline $\begin{array}{l}\text { Radical excision } \\
\text { tumor }\end{array}$ & $100 \%$ & $100 \%$ & $83 \%$ & $P=0.043$ \\
\hline
\end{tabular}


devascularization, and anastomosis, which could define the complexity rating for every procedure. They also concluded that, for different laparoscopic colon procedures, different skills can be learned sequentially if patients are chosen judiciously. Therefore, not only the number of procedures but also the difficulty grade of the intervention will play an important role in the buildup of the learning process for a specific surgeon or team. They advised for one to obtain extensive experience in laparoscopic right colon mobilization and sigmoid colon mobilization before performing left colon resections, low anterior resections, and APRs laparoscopically.

In evaluating the learning curve in laparoscopic colorectal surgery, Tekkis et al. [8] used a multidimensional analysis of the learning curve and compared outcomes between right-sided vs. left-sided colonic resections. They concluded, regarding right-sided and left-sided colonic resections, that it is possible to perform more difficult laparoscopic operations with increased experience without an influence on postoperative morbidity; also, body mass index is a predictive factor in conversion rates and operating time is reduced with experience gained by the surgeon.

Senagore et al. [9] analyzed the learning curve in three consecutive groups of 20 patients and, as expected, found that not only did the number of complications and conversions decreased with experience but more complex surgical resections were also performed with greater ease as surgeons gained more experience. Therefore, it seems important to define the difficulty or complexity grade of a relatively new procedure like the laparoscopic TME.

In the present study, according to the VAS for difficulty, surgeons found the male pelvic anatomy more difficult to operate; however, this was not expressed in terms of a longer operating time, more blood loss, or fewer radical resections. Lower tumors were more difficult to operate when reviewing the operating time, blood loss, VAS for difficulty rewarded by the surgeon, and in the amount of radical resection. Only less blood loss was seen in patients with a BMI of 25-30; operating time, VAS for difficulty rewarded by the surgeon, and oncological radicality of the procedure were comparable for patients with a BMI of 2530 and a $\mathrm{BMI}>30$. Preoperative radiation therapy was found to be accompanied by a longer operating time, more blood loss, a higher VAS for difficulty rewarded by the surgeon, and less radical resections of the tumor.

Not only to advise in the proper way to start, and in an attempt to standardize the learning curve, but also to stratify the different procedures involved in rectal resections to make future study results more comparable, we categorized laparoscopic rectal surgery into three groups of patients, according to their technical difficulty grade: "low-score patients" (scores 6-11), "medium-score patients" (scores
12-24), and "high-score patients" (scores 25-37). We found that there was a significant association between length of surgery, blood loss, VAS for difficulty rewarded by the surgeon, including oncological radicality of the procedure, and the low, medium, and high technical difficulty grade scores for patients, indicating that the score is representative for the technical difficulty grade experienced by the surgeon in the present study.

When reviewing some of the major literature on learning curves in colorectal surgery, the mean number of operations needed within a learning curve is approximately 45 [2-5]. We would therefore suggest for the surgeon to have performed at least 25 operations in the easy technical difficulty grade scores (6-11) and, if the surgeon is confident, to move on to the medium technical difficulty grade scores (12-24). After approximately 25 more operations, the learning curve should be mostly completed and the surgeon could consider moving on to patients with high technical difficulty scores (25-37).

Realizing that the results of the present study were obtained by a surgeon and surgical team who had experience with laparoscopic surgery, the results may have therefore been biased due to their experience. However, we still think that it is important to introduce a technical difficulty grade in the future to better facilitate and standardize teaching purposes, self evaluation, and ultimately patient safety, also for adequate stratification of results in future studies regarding laparoscopic rectal procedures.

We found that the technical difficulty grade score system in the present pilot study could preoperatively predict the difficulty grade of laparoscopic rectal surgery. Surgeons and residents still within their learning curve for laparoscopic TMEs could therefore preoperatively select patients with less difficult procedures and slowly move forward to more complex procedures in a controlled fashion. The present study is a single-institution pilot study; therefore, larger studies need to be conducted for more definite results.

Open Access This article is distributed under the terms of the Creative Commons Attribution NonCommercial License which permits any noncommercial use, distribution, and reproduction in any medium, provided the original author(s) and source are credited.

\section{References}

1. Goligher JC (1984) Surgery of the anus, rectum and colon. Baillière Tindall, London

2. Dagash H, Chowdhury M, Pierro A (2003) When can I be proficient in laparoscopic surgery? A systematic review of the evidence. J Pediatr Surg 38:720-724 
3. Simons AJ, Anthone GJ, Ortega AE, Franklin M, Fleshman J, Geis WP, Beart RW Jr (1995) Laparoscopic-assisted colectomy learning curve. Dis Colon Rectum 38:600-603

4. Bennett CL, Stryker SJ, Ferreira MR, Adams J, Beart RW Jr (1997) The learning curve for laparoscopic colorectal surgery. Preliminary results from a prospective analysis of 1194 laparoscopic-assisted colectomies. Arch Surg 132:41-44

5. Dincler S, Koller MT, Steurer J, Bachmann LM, Christen D, Buchmann P (2003) Multidimensional analysis of learning curves in laparoscopic sigmoid resection: eight-year results. Dis Colon Rectum 46:1371-1378
6. Geis WP, Coletta AV, Verdeja JC, Plasencia G, Ojogho O, Jacobs M (1994) Sequential psychomotor skills development in laparoscopic colon surgery. Arch Surg 129:206-212

7. Geis WP, Coletta AV, Jacobs M, Placensia G, Kim HC (1994) Benefits of complexity scales in laparoscopic colectomy. Int Surg 79:230-232

8. Tekkis PP, Senagore AJ, Delaney CP, Fazio VW (2005) Evaluation of the learning curve in laparoscopic colorectal surgery: comparison of right-sided and left-sided resections. Ann Surg 242:83-91

9. Senagore AJ, Luchtefeld MA, Mackeigan JM (1995) What is the learning curve for laparoscopic colectomy. Am Surg 61:681-685 